\title{
Is Lipid Management Effective for All Stages of CKD?
}

\author{
Elaine $\mathrm{Ku}^{\mathrm{a}}$ Vito Campese ${ }^{\mathrm{b}}$ \\ a University of California San Francisco, San Francisco, Calif., and ${ }^{b}$ Keck School of Medicine, University of Southern \\ California, Los Angeles, Calif., USA
}

\section{Key Words}

Chronic kidney disease $\cdot$ Dyslipidemia $\cdot$ Statin $\cdot$

Cardiovascular disease $\cdot$ Progression of renal disease

\begin{abstract}
Patients with chronic kidney disease (CKD) suffer from high rates of cardiovascular morbidity and mortality. The general approach to traditional cardiovascular risk factor modification via dyslipidemia control has not been thoroughly tested in patients with all stages of CKD. In this article, we review the evidence for statin therapy in patients at various stages of CKD, including patients with CKD on dialysis and patients with kidney transplants.

Copyright $\odot 2013$ S. Karger AG, Basel
\end{abstract}

\section{Introduction}

Patients with chronic kidney disease (CKD) suffer from an increased risk of cardiovascular complications. The etiology of this higher than expected cardiovascular morbidity and mortality has been attributed to a multitude of factors including accelerated atherosclerosis, arteriosclerosis, early development of left ventricular hypertrophy [1], abnormalities of calcium/phosphorus/ parathyroid hormone metabolism [2] and chronic anemia [3]. Although there is solid evidence in the general population that lipid modification lowers cardiovascular risk $[4,5]$, the benefit of lipid management in patients with CKD has only been recently tested.

While many patients with CKD have traditional risk factors for cardiovascular disease (CVD) including hypertension, diabetes, and older age, patients with CKD also have nontraditional cardiovascular risk factors, including the presence of a chronic inflammatory state, increased oxidative stress, activation of the renin-angiotensin-aldosterone system, elevated lipoprotein, homocysteine, and fibrinogen levels, and an overactive sympathetic nervous system [6-8]. The presence of CKD itself is also thought to be a risk factor for CVD in itself [9].

The pattern of dyslipidemia in patients with CKD differs from that of the general population. Patients with CKD often suffer from significant hypertriglyceridemia due to reduced enzymatic processing of triglycerides [10]. During the CKD phase of disease prior to dialysis, patients typically have elevated low-density lipoprotein (LDL) and low high-density lipoprotein (HDL) levels, but once patients are on dialysis, LDL levels frequently fall due to decreased conversion of intermediate-density lipoprotein (IDL) to LDL [10]. As a consequence, elevated levels of LDL precursors, including IDL, are found in circulation. Unfortunately, such precursors are thought to be more atherogenic than LDL and predispose patients to accelerated atherosclerosis [10]. In patients with significant proteinuria and nephrotic syndrome, lipid abnormalities are also pronounced and a significant elevation of LDL is seen [11]. Whether traditional lipid-lowering agents such

\section{KARGER}

Fax +4161306 1234

E-Mail karger@karger.ch

www.karger.com (c) 2013 S. Karger AG, Basel

0253-5068/13/0353-0026\$38.00/0

Accessible online at:

www.karger.com/bpu
Vito M. Campese, MD

Keck School of Medicine at USC

2020 Zonal Avenue

Los Angeles, CA 90033 (USA)

E-Mail campese@usc.edu 
as statins are effective in the setting of an atherogenic environment in the CKD population has been questioned.

In addition to the potential cardiovascular benefits of lipid control, statins have also been found to potentially retard the progression of renal disease in patients with CKD. Mesangial cells in the kidney are thought to have LDL receptors that facilitate the uptake of oxidized LDL, which has cytotoxic effects and triggers both mesangial matrix expansion and an undesirable inflammatory process [12]. In addition, uptake of LDL by macrophages in the kidney are also thought to trigger macrophage transformation to foam cells, leading to increased cytokine release and inflammation, which then subsequently recruits more macrophages and amplifies an inflammatory process [13]. The accumulation of lipoproteins in the kidney may also contribute to the pathogenesis of renal disease progression [14]. Thus, statins could potentially retard the progression of kidney disease by decreasing endothelial damage, improving cardiac function and renal blood flow to the kidneys, and reducing glomerulosclerosis in the kidney [15-17].

\section{Cardiovascular Outcomes and Statins}

Cardiovascular Outcomes in CKD (Stage 1-5) Patients

Patients with CKD, even prior to initiation of dialysis, are at high risk of cardiovascular complications [9]. To modify this risk, statin treatment is frequently considered, but can statins improve cardiovascular outcomes in the CKD population?

One of the major studies of the effects of statins on cardiovascular outcomes was the Treating to New Targets (TNT) trial [18]. In this trial, 10,001 patients with coronary artery disease were randomized between 1998 and 1999 to either atorvastatin 10 or $80 \mathrm{mg}$ daily after a washout period of 1 week (if not previously on a statin) or 8 weeks (if previously on a statin), followed by a 8-week run-in period with atorvastatin $10 \mathrm{mg}$ daily. In this study, the majority of patients were male and white and had a history of coronary heart disease. The mean estimated glomerular filtration rate (eGFR) of participants in the study was $65.6 \mathrm{ml} / \mathrm{min} / 1.73 \mathrm{~m}^{2} .32 \%$ of patients had evidence of CKD at baseline, defined as an eGFR $<60 \mathrm{ml} /$ $\min / 1.73 \mathrm{~m}^{2}$. A secondary analysis of the data showed that after a median follow-up of 5 years, $11.3 \%$ of patients with CKD experienced a major cardiovascular event, compared to $8.6 \%$ of patients with normal eGFR, confirming a significantly higher cardiovascular risk in the CKD population. However, high-dose atorvastatin at 80 mg daily resulted in a $32 \%$ reduction in cardiovascular events in CKD patients and 15\% reduction in patients without CKD when compared to patients treated with atorvastatin $10 \mathrm{mg}$ daily. The study thus indicates that the treatment of dyslipidemia in CKD patients should be more intensive than in patients with normal kidney function, and a lower target LDL should be applied in these patients. Achieving these goals with higher doses of lipidlowering drugs in the past has been considered 'dangerous' because of fear of adverse events in such populations. However, the TNT study participants with CKD did not experience any increased rate of adverse events when compared with patients with normal kidney function, suggesting that high-dose statin therapy is safe in the CKD population.

In the Study of Heart and Renal Protection (SHARP) trial, 9,270 patients with kidney disease (including nondialysis and dialysis patients) were randomized to ezetimibe plus simvastatin versus placebo. Patients in this study experienced a relative risk reduction in a major atherosclerotic event reduction of $17 \%$ (HR $0.83,95 \%$ confidence interval 0.74-0.94, $\mathrm{p}=0.0021$ ) [19]. Again, few adverse effects were noted with statin therapy. However, the relative contribution of ezetimibe to cardiovascular risk reduction outside of its effects on lowering LDL levels is unclear.

Palmer et al. [20] conducted a meta-analysis of over 50,000 patients with all stages of CKD enrolled in various statin trials. According to their meta-analysis, patients with CKD were found to have significant all-cause mortality benefit from statin therapy, but patients on dialysis or post kidney transplant did not benefit from statin therapy. The average dosing in these trials was the equivalent of simvastatin $20 \mathrm{mg}$ daily. The authors note a reduction of cardiovascular event and all-cause mortality by onefourth to one-fifth.

Another large meta-analysis conducted by Upadyhay et al. [21] more recently also favored the use of lipid-lowering therapy for the reduction of cardiovascular mortality and all-cause mortality. This large meta-analysis included 18 trials, 5 of which were randomized controlled trials and 13 of which included subgroup analyses of patients with CKD. The majority of the trials only used statins as main therapy for dyslipidemia, although 2 trials did use statins with ezetimibe, a cholesterol absorption inhibitor. This meta-analysis, again, showed significant benefit with statin use in terms of cardiac mortality, cardiovascular events, and myocardial infarction (MI).

Currently, the evidence available in major statin trials supports a benefit in cardiovascular outcomes in patients with CKD stages 1-5 who are treated with statins. 


\section{Cardiovascular Outcomes in Dialysis Patients}

The presumption that statin therapy will improve cardiovascular outcomes in patients on hemodialysis who are at high risk for cardiovascular events has been tested in several large randomized trials. The first trial to examine this question was conducted in Germany with patients 18-80 years old who had been on dialysis for less than 2 years [22]. The trial randomized 1,255 patients to atorvastatin $20 \mathrm{mg}$ daily versus placebo, and the composite primary endpoint was death from cardiac causes, nonfatal MI, and nonfatal or fatal stroke. Secondary endpoints included death from all causes, all cardiac events combined, and all cerebrovascular events combined. LDL levels were lowered by $42 \%$ during the trial, but unfortunately, there was no difference in the primary endpoint.

The same study group subsequently conducted a post hoc analysis and stratified outcomes by lipid level [23]. Patients who had LDL levels $>145 \mathrm{mg} / \mathrm{dl}$ prior to initiation of statin therapy were found to have a reduction of cardiovascular events by $31 \%$ and a reduction in cardiovascular deaths by $42 \%$. The authors concluded that statin therapy should be considered in patients on hemodialysis with an $\mathrm{LDL}>145 \mathrm{mg} / \mathrm{dl}$. However, the results of this study should be interpreted with some caution, given that this is a post hoc analysis of a study not originally designed or powered to stratify differences in outcome by LDL tier.

A second large randomized double-blinded, placebocontrolled trial, A Study to Evaluate the Use of Rosuvastatin in Subjects on Regular Hemodialysis (AURORA), was conducted between 2003 and 2004 internationally and included dialysis patients between the ages of 50 and 80 who had been on dialysis for less than 3 months [24]. Patients were excluded if they had been on a statin in the last 6 months, had cancer, hypothyroidism, active liver disease, or life expectancy less than 1 year. Patients were randomized to rosuvastatin $10 \mathrm{mg}$ versus placebo with a mean follow-up of 3.2 years. The primary endpoint was time to death from any cardiovascular cause, nonfatal MI, or nonfatal stroke. The secondary endpoint included death from any cause and individual cardiac or vascular events. Although by 3 months, rosuvastatin lowered LDL by $42.9 \%$ compared to baseline LDL levels, no significant difference was noted in the primary or secondary endpoints. Again, this was a disappointing outcome in another large randomized trial showing the lack of benefit to lipid lowering in hemodialysis patients. After AURORA was published, some experts suggested that earlier initiation of statin therapy is needed for cardioprotection, as the burden of cardiac disease is likely already established by the time hemodialysis is initiated
[25]. A post hoc analysis of the 731 diabetic patients enrolled in AURORA did demonstrate a $16 \%$ nonsignificant reduction in the composite primary endpoint, but a $32 \%$ significant reduction in cardiovascular events in diabetic patients [23]. The authors suggest that in hemodialysis patients with diabetes, statin therapy may be effective in reducing cardiac events.

The largest trial to date on the effect of statins in CKD was the SHARP trial conducted in 9,270 patients with either creatinine $>1.5 \mathrm{mg} / \mathrm{dl}$ in men or women $>1.7 \mathrm{mg} / \mathrm{dl}$ or on hemodialysis or peritoneal dialysis [19]. Patients were excluded if they had a definitive history of MI, chronic liver disease, previously on statins, or active inflammatory muscle disorder. Patients were randomized to either placebo, simvastatin $20 \mathrm{mg}$ daily, or simvastatin (20 mg) with ezetimibe $(10 \mathrm{mg})$. Among patients not on dialysis, the average eGFR was $26 \mathrm{ml} / \mathrm{min} / 1.73 \mathrm{~m}^{2}$. The primary outcome was first atherosclerotic event defined as nonfatal MI, coronary death, nonhemorrhagic stroke, or any arterial revascularization procedure. The LDL levels were lowered on average by $0.85 \mathrm{mmol} / \mathrm{l}$ over 5 years and the authors suggest a 19\% reduction in major atherosclerotic events with each $1-\mathrm{mmol} / \mathrm{l}$ reduction in LDL in both dialysis and nondialysis requiring CKD. A significant reduction in nonhemorrhagic stroke and arterial revascularization procedures was noted in patients treated with simvastatin and ezetimibe, but there was a nonsignificant reduction in coronary death and nonfatal MI. The trial was not powered to detect differences in individual outcomes. No difference in effect was noted between patients on dialysis and not on dialysis. The authors suggest that their results differ from the previous 4D and AURORA trials because of a difference in their definition of primary outcome (vascular deaths, which were the focus of previous trials, and a larger number of nonfatal atherosclerotic events) in SHARP.

\section{Cardiovascular Outcomes in Posttransplant Patients}

There have been few studies looking at the effect of lipid modification in patients post kidney transplant. Due to the use of immunosuppressants, the risk of drug interactions and potential adverse effects such as rhabdomyolysis are concerns that tend to dissuade physicians from more intensive use of statins.

Only one major trial, Assessment of Lescal in Renal Transplant (ALERT), has been conducted to date in patients with kidney transplant [26]. In this randomized, double-blind, placebo-controlled trial, 2,102 post kidney transplant patients were randomized to either fluvastatin therapy versus placebo with a mean follow-up of 5.1 years. 
Patients were initially started on fluvastatin $40 \mathrm{mg}$ daily, then the dose was doubled after approximately 2 years. Fluvastatin lowered the mean LDL levels in patients by $32 \%$. The primary endpoint was occurrence of a major cardiovascular event, and secondary endpoints included all-cause mortality, doubling of serum creatinine, or graft loss [27]. Overall, there were fewer cardiac deaths and nonfatal MI in transplant patients treated with fluvastatin. There was no difference in graft loss or doubling of serum creatinine between the fluvastatin and placebo arm. The authors concluded that statin therapy in posttransplant patients may be effective for cardioprotection, but not for renoprotection.

\section{Statins and Renal Outcomes}

There has been long-standing interest in strategies that may slow the progression of disease in patients with CKD. Low protein diet [28], aggressive control of hypertension $[28,29]$, and other various treatment strategies in large-scale trials have all failed to demonstrate any benefit in slowing the progression of kidney disease. What, then, is the role of lipid management in the progression of CKD?

A meta-analysis of 13 small studies conducted by Fried et al. [16] examined the relationship between statin use and progression of renal dysfunction; in this meta-analysis, there was a slightly slower decline in renal function in patients who were treated with lipid-lowering agents, although the etiology of this observed renoprotective effect was unknown. Tonelli et al. [30] subsequently performed a post hoc analysis of a large trial of patients with a history of previous MI randomized to placebo versus pravastatin. This trial enrolled patients with CKD, although the majority of patients included in this post hoc analysis had only mild CKD (GFR 60-89.9 $\mathrm{ml} / \mathrm{min}$ / $1.73 \mathrm{~m}^{2}$ ). A $34 \%$ reduction in loss of renal function was noted in patients with CKD who were treated with pravastatin, although the absolute reduction in the loss in renal function was very small. Patients with moderate to severe CKD and with significant proteinuria were found to derive a greater renoprotective effect than those with mild CKD or with no proteinuria.

In another meta-analysis of 13 randomized trials involving close to 4,000 patients randomized to rosuvastatin, an upward trend in eGFR was noted with longterm statin therapy, but the benefit was not uniform amongst all patients and found to be more significant in African-American patients with CKD [31]. The results of

Is Lipid Management Effective for All

Stages of CKD? these meta-analyses and post hoc analysis was replicated in a randomized controlled trial conducted by Bianchi et al. [32] that was designed to study the effects on atorvastatin and renal function. In this study, 56 patients with CKD were randomized to either atorvastatin versus placebo therapy. The creatinine clearance of patients who were not treated with atorvastatin was noted to decrease significantly compared to those patients treated with atorvastatin.

The SHARP trial, conducted in 9,270 patients with either creatinine $>1.5 \mathrm{mg} / \mathrm{dl}$ in men or women $>1.7 \mathrm{mg} / \mathrm{dl}$ or on hemodialysis or peritoneal dialysis, also examined the effect of statin therapy on the progression of kidney disease [19]. Disappointingly, patients allocated to simvastatin and ezetimibe did not show a decreased incidence in ESRD or time to doubling of serum creatinine. A large meta-analysis that included 50 trials and over 30,000 patients looking at the effects of statins in CKD also found no renoprotective effect [33].

Are all statins, then, potentially beneficial in slowing the progression of renal disease? The answer currently is unclear, as few randomized trials have compared the effect of various statins on renal disease progression. The Prospective Evaluation of Proteinuria and Renal Function in Diabetic Patients with Progressive Renal Disease (PLANET I and II trials) compared the effect of rosuvastatin versus atorvastatin on proteinuria and found that rosuvastatin had no effect of proteinuria and potentially accelerated the rate of renal function loss. In this trial, atorvastatin did not have significant benefit on renal function, but was effective in decreasing proteinuria [34]. In contrast, a meta-analysis of 9 randomized controlled trials focusing on atorvastatin did find a significant benefit to renal function [35]. Thus, whether all statins are equally beneficial in their renoprotective effect is unclear.

\section{Conclusion}

CKD leads to major cardiovascular morbidity and mortality. Most large randomized trials involving statin therapy in patients with CKD have shown few adverse effects with statin use and significant cardioprotection. Statins should be initiated early in the course of CKD to reduce CVD risk, as the benefits of initiation of therapy, once patients are on dialysis, is debatable. The renoprotective effect of statins slowing the progression of disease remains to be determined in both patients with CKD and posttransplant and it may be related to the amount of proteinuria. 


\section{References}

1 Sarnak MJ: Cardiovascular complications in chronic kidney disease. Am J Kidney Dis 2003;41:11-17.

2 Khan AM, Chirinos JA, Litt H, Yang W, Rosas SE: FGF-23 and the progression of coronary arterial calcification in patients new to dialysis. Clin J Am Soc Nephrol 2012, E-pub ahead of print.

3 Parfrey PS: Why does the treatment of anaemia not improve cardiac outcomes in CKD? Nat Rev Nephrol 2012, E-pub ahead of print.

$\checkmark 4$ Downs JR, Clearfield M, Weis S, et al: Primary prevention of acute coronary events with lovastatin in men and women with average cholesterol levels: results of AFCAPS/ TexCAPS. Air Force/Texas Coronary Atherosclerosis Prevention Study. JAMA 1998 279:1615-1622.

5 The Scandinavian Simvastatin Survival Study (4S): Randomised trial of cholesterol lowering in 4,444 patients with coronary heart disease. Lancet 1994;344:1383-1389.

-6 Park J: Cardiovascular risk in chronic kidney disease: role of the sympathetic nervous system. Cardiol Res Pract 2012;2012:319432.

7 Nitta K: Clinical assessment and management of dyslipidemia in patients with chronic kidney disease. Clin Exp Nephrol 2012;16: 522-529.

$>8$ Kendrick J, Chonchol MB: Nontraditional risk factors for cardiovascular disease in patients with chronic kidney disease. Nat Clin Pract Nephrol 2008;4:672-681.

9 Go AS, Chertow GM, Fan D, McCulloch CE, Hsu CY: Chronic kidney disease and the risks of death, cardiovascular events, and hospitalization. N Engl J Med 2004;351: 1296-1305.

$>10$ Kwan BC, Kronenberg F, Beddhu S, Cheung AK: Lipoprotein metabolism and lipid management in chronic kidney disease. J Am Soc Nephrol 2007; 18:1246-1261.

11 Vaziri ND: Dyslipidemia of chronic renal failure: the nature, mechanisms, and potential consequences. Am J Physiol Renal Physiol 2006;290:F262-272.

12 Rovin BH, Tan LC: LDL stimulates mesangial fibronectin production and chemoattractant expression. Kidney Int 1993;43:218225.

13 Oda H, Keane WF: Recent advances in statins and the kidney. Kidney Int Suppl 1999;71:S2-S5.
14 Samuelsson O, Mulec H, Knight-Gibson C, et al: Lipoprotein abnormalities are associated with increased rate of progression of human chronic renal insufficiency. Nephrol Dial Transplant 1997;12:1908-1915.

15 Athyros VG, Mikhailidis DP, Papageorgiou AA, et al: The effect of statins versus untreat ed dyslipidaemia on renal function in patients with coronary heart disease. A subgroup analysis of the Greek atorvastatin and coronaryheart disease evaluation (GREACE) study. J Clin Pathol 2004;57:728-734.

16 Fried LF, Orchard TJ, Kasiske BL: Effect of lipid reduction on the progression of renal disease: a meta-analysis. Kidney Int 2001;59: 260-269.

17 O’Donnell MP, Kasiske BL, Kim Y, Schmitz PG, Keane WF: Lovastatin retards the progression of established glomerular disease in obese Zucker rats. Am J Kidney Dis 1993;22: 83-89.

18 Shepherd J, Kastelein JJ, Bittner V, et al: Intensive lipid lowering with atorvastatin in patients with coronary heart disease and chronic kidney disease: the TNT (Treating to New Targets) study. J Am Coll Cardiol 2008; 51:1448-1454.

19 Baigent C, Landray MJ, Reith C, et al: The effects of lowering LDL cholesterol with simvastatin plus ezetimibe in patients with chronic kidney disease (Study of Heart and Renal Protection): a randomised placebocontrolled trial. Lancet 2011;377:2181-2192.

20 Palmer SC, Craig JC, Navaneethan SD, Tonelli M, Pellegrini F, Strippoli GF: Benefits and harms of statin therapy for persons with chronic kidney disease: a systematic review and meta-analysis. Ann Intern Med 2012; 157:263-275.

21 Upadhyay A, Earley A, Lamont JL, Haynes S, Wanner C, Balk EM: Lipid-lowering therapy in persons with chronic kidney disease: a systematic review and meta-analysis. Ann Intern Med 2012;157:251-262.

22 Wanner C, Krane V, Marz W, et al: Atorvastatin in patients with type 2 diabetes mellitus undergoing hemodialysis. N Engl J Med 2005;353:238-248.

23 Marz W, Genser B, Drechsler C, et al: Atorvastatin and low-density lipoprotein cholesterol in type 2 diabetes mellitus patients on hemodialysis. Clin J Am Soc Nephrol 2011;6: 1316-1325.

-24 Fellstrom BC, Jardine AG, Schmieder RE, et al: Rosuvastatin and cardiovascular events in patients undergoing hemodialysis. N Engl J Med 2009;360:1395-1407.
25 Athyros VG, Tziomalos K, Karagiannis A, Mikhailidis DP: Statins and cardiovascular events in patients with end-stage renal disease on hemodialysis. The AURORA results suggest the need for earlier intervention. Curr Vasc Pharmacol 2009;7:264-266.

26 Holdaas H, Fellstrom B, Jardine AG, et al: Effect of fluvastatin on cardiac outcomes in renal transplant recipients: a multicentre, randomised, placebo-controlled trial. Lancet 2003;361:2024-2031.

-27 Fellstrom B, Holdaas H, Jardine AG, et al: Effect of fluvastatin on renal end points in the Assessment of Lescol in Renal Transplant (ALERT) trial. Kidney Int 2004;66:15491555.

28 Levey AS, Adler S, Caggiula AW, et al: Effects of dietary protein restriction on the progression of advanced renal disease in the Modification of Diet in Renal Disease Study. Am J Kidney Dis 1996;27:652-663.

29 Wright JT Jr, Bakris G, Greene T, et al: Effect of blood pressure lowering and antihypertensive drug class on progression of hypertensive kidney disease: results from the AASK trial. JAMA 2002;288:2421-2431.

30 Tonelli M, Moyé L, Sacks FM, et al, for the Cholesterol and Recurrent Events (CARE) Trial Investigators: Effect of pravastatin on loss of renal function in people with moderate chronic renal insufficiency and cardiovascular disease. J Am Soc Nephrol 2003; 14:1605-1613.

31 Vidt DG, Harris S, McTaggart F, Ditmarsch M, Sager PT, Sorof JM: Effect of short-term rosuvastatin treatment on estimated glomerular filtration rate. Am J Cardiol 2006;97: 1602-1606.

32 Bianchi S, Bigazzi R, Caiazza A, Campese VM: A controlled, prospective study of the effects of atorvastatin on proteinuria and progression of kidney disease. Am J Kidney Dis 2003;41:565-570.

-33 Strippoli GF, Navaneethan SD, Johnson DW et al: Effects of statins in patients with chronic kidney disease: meta-analysis and metaregression of randomised controlled trials. BMJ 2008;336:645-651.

34 PLANET I and II: Atorvastatin beats rosuvastatin for protecting kidneys in diabetic and nondiabetic patients. 2010. Accessed 10/27/2012 at http://www.theheart.org/article/1095269.do.

35 Takagi H, Umemoto T: A meta-analysis of randomized trials for effects of atorvastatin on renal function in chronic kidney disease. Int J Cardiol 2011;152:242-244. 